Thought rapidity and time reaction showed the same paralysis. With the aid of a battery and a clock marking the parts of a second the time was measured from the moment an object came into the field of vision to its record on a dial by the pressure on a button. This, in parts of a second, showed both the rapidity of sight and brain power of registration. If in health such impressions could be recorded in a fourth of a second from the time it was seen, after the use of spirits this time was lengthened to one or more seconds. Another experiment to show thought rapidity was to repeat words or short sentences uttered by others and registered in the same way by an electric clock. The health standard having been found, the action of spirits literally doubled the time in these cases. Numerous experiments involving reason and discrimination were made, one in which the person was asked to pick out pictures of animals and name them from a group in sight. After the use of spirits the time required was 40 per cent. longer, showing impairment both of the senses and the power of reasoning. In some experiments which I have made on simple mathematical calculations the percentage of mistakes was from 15 to 20 per cent. greater after spirits were used than before. Persons blindfolded and given tasks requiring consciousness, reasoning and correct thought were found to show very marked change and palsy after spirits were used.

These experiments may be carried on in a great variety of ways; having first found the normal condition of the person, the deviation from this can be ascertained and measured with great accuracy. During the past year Kraepelin has published some new studies. in which he has been able to recognize and measure the effects of half an ounce of ethylic spirits. This would be equivalent to an ordinary glass of one or one and onehalf ounces of the common strong drink. One ounce of spirits is the ordinary equivalent of two and one-half ounces of whiskey containing from 40 to 50 per cent. of alcohol. These experiments are very significant, and seem to point out conclusively that alcohol in these doses is distinctly a paralyzer. In some work done in this country along this line where a single dram of alcohol is used, the effect on the sight and hearing was transient, but the effect on the higher brain was measurable. The diffieulty in having a personal equation or normal standard from which to judge is a serious one. I have been able to point out in two cases very marked depression of the senses and brain activities from two drams of alcohol, but not having any knowledge of the person, the value of these experiments were limited.

These experimental studies are supported by. clinical experience and fully illustrated in every person intoxicated by alcohol. The observations of a number of persons, including the writer, show that in all toxic states from spirits there is the same palsy of the senses and of muscular power, depressed heart's action and slowing up of the mental activities of the brain. Hence the researches of Kraepelin confirm these facts and show conclusively that the paralyzing action of alcohol begins at a very early period and from a very small quantity of spirits. It is difficult to believe that it has any other action on the nerve centers or protoplasm of the body than that of a narcotic and paralyzant.

Rice cooked with milk furnishes the most easily digested food with the largest amount of nutriment contained in moderate bulk.-New York Medical Journal.
THE LEGITIMATE THERAPEU'IIC USES OF ALCOHOL.*

O. T. OSBORNE, M.A., M.D.

Professor of Materia Medica and Therapeutics at Yale Medical School.

NEW HAVEN, CONN.

In discussing this topic it is best to first state just what we have the right to expect from alcohol as a medicine. The desired action from a dose of whiskey or brandy or its equivalent is a warming of the surface of the body, an increased pulse tension, a moistening of the dry skin, absence of nausea, absence of much flushing of the face, absence of throbbing in the head, unless a case of syncope is treated by a large dose, when many of these primary undesired symptoms might occur. In giving alcohol continuously the dose should be regulated by the signs. If it causes flushing of the face, dry skin, dry tongue, a throbbing, bounding pulse, even temporarily, and the odor of alcohol is on the breath, the dose is too large.

Alcohol is by no means a cure-all, and, undoubtedly, in the past much harm has been done by its. indiscriminate use. Perhaps, even to-day, it is us more frequently and more freely than its physiologic action warrants, and it should never be prescribed unless there is some definite indication for it. Indications for the use of alcohol may be divided into:

1. Local.

2. To stimulate the heart.

3. As a food.

4. To increase the appetite and aid digestion.

5. To relieve acute internal congestion.

6. To dilate and relax the peripheral circulation.

7. To produce sleep.

8. To combat poisons in the system.

\section{THE LOCAL USE OF ALCOHOL.}

'The stronger preparations of alcohol may be applied to any part of the surface of the body as an astringent to stop secretion and to harden the tissues. For this purpose it may be used to harden the nipples to prepare for suckling, to stop profuse perspiration of the axillie or feet, to prevent bed-sores, and wcaker alcoholic preparations may enter into the composition of hair tonics to prevent too much sebaceous secretion and oiliness of the hair. Strong alcohol is hemostatic to capillary onzing. Absolute alcohol more or less constantly applied to the surface over a eginning boil, as in the ear for instance, may prevent its culmination and abort it. In concentrated form it will act as a rubefacient while being rubbed on the skin, but if allowed to evaporate it will cool the surface of the body. If diluted it evaporates more slowly and the cooling is prolonged, hence it is often used to reduce temperature. If the surface of the body is sponged with warm alcohol it is sometimes very soothing, quieting restlessness and producing mental calmness in a feverish patient. Probably a large part of the advantage of the different tinctures and extracts used for sprains, bruises, ctc., is due to the alcohol which they contain. Alcohol diluted somewhat, depending on the thinness of the skin, applied on gauze or lint or lintine and covered with rubber tissue or other inpervious dressing and changed every six hours, will remove the swelling

* Read at the Fifty-fourth Annual Session of the American Medical Association, in the Section on Materia Medica, I'barmacy and Therapeutics, and approved for publication by the Fxecutive Davis, Jr. 
and pain from a sprain and aid in the cure or prevention of synoritis, myositis or inflammation of the tendons quicker than most any other treatment we can institute. Diluted alcohol, one to four or five parts of water, or weaker, makes a good astringent and antiseptic mouth wash, hardening the gums and sweetening the breath. Flannel or towels wrung out of very hot water sprinkled with alcohol and placed on the abdomen or any other part of the body and changed every fifteen minutes for an hour or two causes a splendid rubefacient and counter-irritant action. $A$ brick heated in the oven, wrapped with three or four layers of flannel dipped in boiling water and allowed to drain, and then a considerable amount of alcohol poured on it, placed beside a warmly covered patient in bed, will produce a splendid alcohol steam bath. Locally applied to the back in this form it is very efficient in the treatment of lumbago.

In the form of alcohol brandy is especially a carminative and an astringent for diarrhea, and markedly so in the form of blackberry brandy.

\section{TO STIMULATE THE HEART.}

There is a considerable difference of opinion to-day as to the value of alcohol as a cardiac stimulant. Its action on the vascular system, however, is marked and positive. The irritation which strong alcohol in any form causes on the mucous membrane of the throat, esophagus and stomach acts reflexly through the medulla to cause vasoconstriction and, hence, a rise of blood pressure, and increased blood pressure is always a stimulant to the heart. After alcohol is absorbed into the blood, where it circulates as alcohol, it can not, in my opinion, by its physiologic action on the vasomotor centers, on the blood vessels, and the heart, ever be a stimulant, although, as its secondary effect is to dilate the blood paths, this very effect of increasing the peripheral circulation may better the condition of the heart. Through, then, the irritant impact of the alcohol on the mucous membrane the blood pressure is first raised, but when it is absorbed the blood vessels dilate, especially those on the surface of the body. This relieves any spasmodic contraction of the peripheral blood vessels, whether from fear, from pain, or from chilling of the body, and this equalization of the circulation relieves any internal congestion that may be present. By this widening of the blood paths, provided no condition of shock is present, alcohol will relieve the laboring of a weakened heart. If too large doses of alcohol are given this vasodilatation goes on to partial paralysis of the blood vessels, and a weak action of the heart from this cause alone will occur. Hence, in shock where the blood vessels are widely dilated or perhaps paralyzed, either from chloroform or ether narcosis, or from morphin narcosis, or from injury or collapse, alcohol, except for its primary stimulant action in small doses, would do harm and is contraindicated.

The exact action of alcohol on the heart itself is not proved beyond a reasonable doubt. Certain it is that overdoses of alcohol after absorption are depressant to the heart muscle, to the muscle fibers of the blood vessel walls, and to the vasomotor center in the medulla. In sudden cardiac failure the reviving action of alcohol seems to me to be due entirely to the reflex stimulation of the mucous membrane of the throat and stomach. By this stimulation we cause an increased cardiac frequency and a slightly raised blood pressure from the contraction of the arterioles due to the reflex action on the medulla. This action, which occurs before much alcohol has been absorbed, may tide over the condition of acuite cardiac failure, and if not much alcohol has been given there will be no future cardiac or vascular depression. If too much alcohol has been administered and absorbed the result will be to increase the previous depression unless more fresh alcohol is given for its continued stimulant reflex effect. If the previous doses are not too large and are, hence, rapidly burned or eliminated, the effect will be continued stimulation and not depression.

In conditions of high temperature where the heart is very rapid from the stimulation of the fever, from the irritation of the toxins in the blood, and from nervous irritability, alcohol, by quieting the nervous system, dilating the peripheral blood vessels, and possibly combating the toxins in the blood, generally reduces the temperature and slows the pulse. If the dose is properly regulated, this action will be found to be.continuous for good. Too large doses or too frequent administration of alcohol may do one of two things: either stimulate the brain and cause more cxcitation, increasing the temperature by increasing muscular irritability and restlessness and causing enough of its local reflex irritation to make the pulse bounding and full, or, if it is too rapidly absorbed, it will give the symptoms of alcoholic depression on the circulatory system. Hence, in acute cardiac failure to get the stimulant effect of alcohol it must be given concentrated as exemplified by brandy, whiskey, gin, rum and champagne, and in small doses. To keep up this stimulation another dose must be given in from fifteen minutes to half an hour or an hour, depending on the continuousness of the heart weakness, in order to repeat the stimulation of the irritation. As just stated, the close should undoubtedly be small, as the future depression from the absorption would be less than it would from large doses. If the frequency of the dose is such that alcohol piles up in the system and more is given before the previous ingested amount has been burned or climinated, to obtain stimulation it will be necessary to give larger and larger doses, first to overcome the depression caused by the previous alcohol, and also to cause the usual stimulation. As more and more alcohol is alssorbed whether from the stomach or from hypodermic injection, depression, vasomotor dilatation, cardiac weakness, and more or less paralysis of the nervous system are sure to follow. Hence, to combat continuous or persistent cardiac failure or depression, alcohol is the poorest stimulant we can use, and if much is given we are poisoning the system and adding depression to depression that is already there and the treatment is vicious.

In conditions of prolonged fever where alcohol is given no more frequently for any length of time than every three hours, one dose is probably eliminated or burned before the next one is given; and we get the primary stimulation plus the dilatation of the peripheral vessels, which equalizes the circulation and reduces the temperature.

Let me parenthetically say that I have often obtained the same good circulatory results in sthenic fever from small doses of nitroglycerin that $I$ have obtained with alcoho!.

In serious feverish conditions undoubtedly the narcotic effect of alcohol in quieting the patient, who is highly alert to his danger, may be of distinct advantage, as mental worry and anxiety are great disturbers of the 
heart and circulation and should be overcome by any legitimate means.

The only time, then, that I belicve alcohol can meet the indication for a cardiac stimulant is in acute cardiac failure, and then it is a stimulant to the heart only before its ahsorption, by the irritation which it causes the mucous membrane of the mouth, pharynx, esophagus and stomach, or the rectum, if it is given as an enema, or by the irritation of the tissues, if it is given hypodermically.

ALCOFIOL AS A FOOD.

I believe that as a nutriment alcohol is uneconomical to the system and if long used is a positive disadvantage, probably even sugar being better where the amount of food obtainable is too small for the needs of the body. During feverish processes, however, as a nutriment to furnish material for the production of heat to save the tissues, fat and muscles it is of decided advantage and probably has no equal, if in such fevers not enough nutrition is taken. Undoubtedly at the present time many cases of fever are overfed. Whether whiskey or brandy should be given in every case of typhoid fever or diphtheria, or in the feverish debility of tuberculosis, or in other acute infections, is a question that each practitioner must decide for each individual case. Many such cases do well without it; many cases recover only by the use of it.

TO INCREASE THE APPETITE AND DIGESTION.

This is a very questionable indication to mect with alcohol. In occasional cases of neurasthenia, anemia, malnutrition, and inertia of the stomach, with imperfect gastric secretion, a small cocktail before meals may be justifiable and proper, but, rather than select a known alcoholic beverage for this purpose, some bitter dilute tincture will do as well, and will more readily preclude the possibility of acquiring the habit. Late in life some such stimulant to nutrition may be justifiable.

TO RELIEVE ACUTE INTERNAL CONGESTION.

After becoming chilled or getting wet or being subjected to other exposure, as the exposure of exhaustion, hot alcohol in some form combined with a hot general bath, or a hot foot bath and a warm bed, with or without a Dover's powder or quinin, is undoubtedly good treatment as tending to relieve or abort the internal congestion, whether it be nasal, pharyngeal, tonsillar, laryngeal, bronchial, pulmonary, pleuritic, nephritic, arthritic or intestinal.

\section{TO RELAX THE PERIPHERAL CIRCULATION.}

This indication for a vasodilator is present only in gouty or atheromatous conditions of the blood vessels or whenever there is too high blood pressure in the middle and late stages of life. Generally without any doubt this indication is better met by small doses of nitroglycerin, thyroid extract, potassium iodid or small doses of chloral, as well as by causing increased elimination from the skin and intestines. However, many cases occur in which alcohol has been more or less regularly taken and a small amount once or twice a day with the meals, provided the amount is such as not to cause fiushing or a feeling of fullness in the head, may be of positive advantage.

\section{TO PRODUCE SLEEP.}

The use of alcohol for this purpose is only justifiable in exceptionally acute conditions or where the condition is probably not to recur, or in old pcople. A little nightcap of some alcoholic beverage, often, perhaps, beer, perhaps a spirit, diluted so as to prevent much stimulation, which would cause sleepléssness, just sufficient to dilate the abdominal and peripheral vessels and thus to depress the circulation in the brain, will act as a hypnotic. There are many other, perhaps, generally better methods of causing this sleep, but often the association of symptoms and conditions is such in elderly people that it is good treatment to use alcohol.

TO COMBAT POISONS IN THE SYSTEM.

It is difficult to discuss, with any reasonableness, this indication. It has long been stated, but often disputed, that alcohol in large amounts will prevent dire results from the bites of poisonous snakes. If alcohol is given in enormous amounts in the shape of whiskey or brandy the paticnt is soon in the first stage of alcoholic poisoning. Some acute infections in which death scems imminent have been said to have been stopped by enormous doses of alcohol, notably the final toxemia of pneumonias. The antitoxic power of alcohol in the blood against these toxins must be corroborated or disproved in the laboratory. Clinical experience in this line leaves so much room for doubt that the matter is still subject to proof.

\section{DISCUSSION}

ON TIIE PAPERS OF DRS. CROTIYERS AND OSBORNE.

Dr. J. H. Musser, Philadelphia-I use less alcohol in my practice than I did in former years, particularly in fevers. I feel that we are employing a dangerous remedy when we use alcohol, and for moral reasons, as well as because of the doubtful pathologic results, I employ less and less of it. The indications for its use in acute diseases are those found in intoxications as arise in septicemia and for its narcotic properties. Under these circumstances patients seem to be able to stand enormous amounts, $\mathbf{i}$ e., in streptococeus infectiuns, and in that type of typhoid fever seen in the aged-the septicemic type. In typhoid fever in the young, when the disease presents actual septicemic symptoms, I use it, but I prefer to use it in very small doses, and I give large doses only for a short period of time. I believe that if we withhold it and ther. for a short time give it in full doses we may possibly pull the patient through a condition to which he would otherwise succumb. Each year I use less alcohol, particularly in the two diseases in which we were wont to give it frequentlytyphoid fever and pneumonia; here I use it only as an emer. gency remedy and for short periods of time. It is interesting for one to look back over his career and note any changes in his ideas. I am pretty well satisfied that I have more patients recover from typhoid fever now than ever before, and I believe this is largely due to the manner in which $I$ bandle stimulants. Of course, there are two periods in the management of this disease, in my career, the period before the bath and the period since the bath was instituted. In the latter period, in the cold water period, I find that patients get along better without alcohol than they formerly did with alcohol and no baths. Of course, this is to be expected. Moreover, under eircumstances beyond our control baths can not be employed or are contraindicated. In this group of cases I use much less quantities of stimulants with better results-certainly at no disadvantage to my patients-than formerly. I believe I can say that without qualification as to the character of the disease. I think I have seen sicker people who are not put in tubs in typhoid fever in the latter period than in the time before the baths, and yet the mortality is less. I certainly can not attribute the lessened mortality to any. thing else because we do not use other drugs to any extent, and when we do use them it is only as we use alcohol, just as the occasion demands for a brief period and then stop.

DR. J. A. Storck, New Orleans--We ought to try to find out the action of alcohol in carbolic acid poisoning. What is the rationale of the antidotal effect of alcohol in carbolic acid 
poisoning? In a recent meeting of a medical society this question was threshed over, but no definite conclusion was arrived at as to whether its action was due to chemical change or to mere dilution. The fact remains, however, that alcohol is the ideal antidote for carbolic acid poisoning.

DR. W. J. RoBisson, New York-In reference to the statement by many physicians that they are using alcohol less ant less each year, I have no doubt that a good many do use less, but I come across some who think they use less, yet in reality they do not. For instance, a number will give liquid peptonoids and other preparations under the impression that they are not giving alcohol, and yet we know that these things contain from 20 to 25 per cent. of alcohol, so that when a physician gives 8 ounces of liquid peptonoids he gives about 4. ounces of whisky. This is, of course, not true of all physicians, but it is a fact in many instances. In reference to septicemia, especially puerperal septicemia, I do not believe there is any drug equal to alcohol. As to alcohol and carbolic acid, there is a true chemical reaction. 'This is a fact; it is not a simple dilution, because we can not get the same effect with water, even if the latter be used in 100 times the amount. Experiments have been made in this direction on the outside of the body, and it has been shown that alcohol completely neutralizes the carbolic acid, and probably the same effect is obtained in the body.

DR. J. H. MUsser, Philadelphia-I do not bother with panopeptone and such preparations except in cases in which we can not give food by the stomach. It is a class of remedies that 1 consider pernicious.

Dr. A. C. Croftan, Chicago-Few people realize that alcohol has been demonstrated to be a normal product in the disassimilation of sugar. I have done some work along this line. If you take a large quantity of human muscle, or pancreas, for instance, and, under certain technical precautions, distill it with water you will get an appreciable quantity of ethyl alcohol. The knowledge of this would probably horrify the temperance advocates. From a purely physiologic standpoint this may indicate that a small quantity of alcohol is probably a physiologic stimulant.

Dr. S. Sols-Cohen, Philadelphia-Are the sulphates a better antidote to carbolic acid poisoning than alcohol?

Prof. C. S. N. Hallberg, Chicago-The alkali sulphates have always been regarded as the chemical antidotes because the double decomposition forms sulphocarbolates, but alcohol has certain effects which can not be obtained by using the sulphates. In addition to alcohol, camphor has a modifying effect on the carbolic acid, so that their combination, as in spirit of camphor, would probably be more effective.

DR. S. Solis-Conex, Philadelphia-I am one of those who, like Dr. Musser, do not use disguised forms of alcohol, and yet $I$ am conscious that my resort to alcohol in acute febrile affections, and especially in typhoid fever, has been lessening steadily. In fact, I never think of prescribing it, unless some special condition calls for it; in other words, I use it as I would use opium or any other drug, in specified dose for a special purpose.

Dr. T. D. Crotuers, Hartford, Conn.-We have reached a point in experimental researches where certain facts can be demonstrated with a reasonable degree of accuracy. All assertions concerning the action of alcohol on the body should not be accepted to-day, unless they have been subject to some scientific test and eritical examination. The elinical fact that alcohol is an excellent refrigerant in fevers when used externally can be confirmed with great exactness and traced to the rapid water-absorbing property of alcohol. When alcohol is used as a stimulant and tonic, scientific experiment fails to point out the evidence necessary to sustain this assertion, hence such theories are only vague unsupported inferences. While it can be shown that alcohol acts most positively on the heart and vasomotor centers both increasing and slowing up the organ and the circulation of the blood, the effect of this peculiar action is unknown, and until more accurate researches are made we must regard this action as more or less dangerous. Any remedy which disturbs the nutrition and the rhythmic
How of blood through the brain should be used with great caution. The known effects of alcohol on the senses, blood vessels and heart are very limited compared with the unknown, and yet this little knowledge is startling in the clearness with which it points out the corroding action of this agent, together with other effects not yet studied. The toxic action of alcohol, either in small or large quantities, falls most seriously on the blood vessels and heart; beyond this stretches a wide unexplored country. My paper is simply a contribution to the work that is to be done in this direction, and also an indication of how wide the domain of exact studies are.

J). O. T. Osborne, New Haven, Conn.-I wish to emphasize the fact brought out in the paper that we have been using alcohol during all of these years as a cardiac stimulant in fevers, and we come to find out that it never acts as a cardiac stimulant, but that it acts as a vasodilator, and hence is of value in sthenic cases. I have seen nitroglycerin effect the same good results as alcohol. Cirrhosis of the liver caused by overuse of aicohol simply bears out the fact that continued irritation of any organ sooner or later tends to produce connective tissue in that organ.

\section{NASAI, POLYPUS ; ORIGIN AND TREATMENT.* CHAS. H. BAKER, M.D. BAY CITY, MICH.}

'There is a variety of growths within the nasal chambers to which the name polypus has been given, and much confusion has arisen in consequence, both in regard to the origin and frequency of nasal polvpus. Those who restrict the term nasal polypus to the gelatinous form find them comparatively infrequent compared to the total of nasal disorders, while others, like Zuckerkandl, who included posterior turbinate hypertrophies and fibro-adenomata, claim that they occur in every eight or nine autopsies.

It is my purpose to confine this paper to the discussion of the true nasal polypus or gelatinous myxoma, although what I shall say as to their causation will apply largely to the other growths which have been classed as polypi.

\section{ORIGIN.}

True nasal polypus has by different pathologists been classed among the adenomata or the myxomata according to whether one or the other elements predominated in the structure. The prevailing type is that of a pure myxoma with delicate connective tissue stroma, few vessels and made up principally of the characteristic stellate myxomatous cells imbedded in gelatinous intercellular substance, chiefly mucin.

There are certain variations according to location, life history and presence or absence of outside interference or irritations, and many are found with much connective tissue and glandular structure replacing the ordinary mucous contents. The fact that mucous polypi seem occasionally to undergo cystic degeneration indicates that they may be partly glandular in origin, but true cystic degeneration of the mucous polypus does not occur.

By far the majority of polyps spring from the anterior edge of the middle turbinate or from the edges of the hiatus semilunaris; next from the upper and posterior portions of the middle turbinate and, lastly, may be found in the interior of a bullous middle turbinal or about the outlets of the accessory sinuses. Very rarely they have been found in other localities, but when so

* Read at the Fifty-fourth Annual Session of the American Medical Association, in the Section on Laryngology and Otology, and approved for publication by the Executive Committee: Drs. George
L. Richards, O. Joachim and G. V. Woollen. 